\title{
STRONGLY SIMPLY CONNECTED AUSLANDER ALGEBRAS
}

\author{
by IBRAHIM ASSEM and PETER BROWN
}

(Received 23 June, 1995)

Let $k$ be an algebraically closed field. By an algebra is meant an associative finite dimensional $k$-algebra $\Lambda$ with an identity. We are interested in studying the representation theory of $\Lambda$, that is, in describing the category $\bmod \Lambda$ of finitely generated right $\Lambda$-modules. Thus we may, without loss of generality, assume that $\Lambda$ is basic and connected. For our purpose, one strategy consists in using covering techniques to reduce the problem to the case where the algebra is simply connected, then in solving the problem in this latter case. This strategy was proved efficient for representation-finite algebras (that is, algebras having only finitely many isomorphism classes of indecomposable modules) and representation-finite simply connected algebras are by now well-understood: see, for instance [5], [7], [8]. While little is known about covering techniques in the representation-infinite case, it is clearly an interesting problem to describe the representation-infinite simply connected algebras. The objective of this paper is to give a criterion for the simple connectedness of a class of (mostly representationinfinite) algebras.

Let $\Lambda$ be a representation-finite algebra, and $\left\{M_{1}, \ldots, M_{d}\right\}$ a complete set of representatives of the isomorphism classes of indecomposable $\Lambda$-modules. The algebra $A=\operatorname{End}_{\Lambda}\left(\oplus_{i=1}^{d} M_{i}\right)$ is called the Auslander algebra of $\Lambda$ (see [4]). Auslander algebras, introduced first in [3], are characterised by their homological properties, which were useful, for instance, in the development of covering theory [7]. We are interested in the simple connectedness of the Auslander algebra $A$ of $\Lambda$. A related question is the study of the first Hochschild cohomology group $H^{1}(A)$ of $A$ with coefficients in the bimodule ${ }_{A} A_{A}$ (see [9]). The connection between the simple connectedness of an algebra and the vanishing of its first Hochschild cohomology group was explored in [10], [15], [2]. In particular, Skowroński considers in [15] the class of strongly simply connected algebras, that is, the algebras $A$ with the property that each full convex subcategory $C$ of $A$ is simply connected (where $A$ is viewed, as in [7], as a locally bounded $k$-linear category). He proves that $A$ is strongly simply connected if and only if, for each full convex subcategory $C$ of $A$, we have $H^{1}(C)=0$. Further, Happel showed in [11] that a representation-finite algebra $\Lambda$ is simply connected if and only if $H^{1}(A)=0$ where $A$ denotes, as above, the Auslander algebra of $\Lambda$. Our main result characterises the (strong) simple connectedness of Auslander algebras, and generalises the aforementioned result of Happel.

THEOREM. Let $\Lambda$ be a representation-finite algebra, and let $A$ be its Auslander algebra. The following statements are equivalent:

(a) $\Lambda$ is simply connected;

(b) $A$ is simply connected;

(c) $A$ is strongly simply connected;

(d) $H^{1}(A)=0$;

(e) $H^{1}(C)=0$ for each full convex subcategory $C$ of $A$.

Glasgow Math. J. 39 (1997) 21-27. 
The results of Happel and Skowroniski imply respectively the equivalence of (a) and (d), and of (c) and (e). Since (c) clearly implies (b), we just have to show that (a) implies (c), and that (b) implies (a).

This paper is organised as follows. In Section 1, we show that simple modules over a full convex subcategory $C$ of the Auslander algebra $A$ of a representation-directed algebra have projective resolutions similar to those of the simple $A$-modules. Section 2 is devoted to the proof of our main result. We shall use freely, and without further reference, results about the Auslander-Reiten quiver $\Gamma(\bmod \Lambda)$ of an algebra $\Lambda$, and the Auslander-Reiten translations $\tau=D \operatorname{Tr}$, and $\tau^{-1}=\operatorname{Tr} D$ as can be found, for instance, in [4], [14]. For the basic facts about simply connected algebras, we refer the reader to [1], [2], [15].

1. The Auslander algebra of a representation-directed algebra. We shall need the following general considerations. Let $A$ be an algebra, considered as a locally bounded $k$-linear category [7], and $C$ be a full convex subcategory of $A$. For any $a$ in the set of objects $A_{0}$ of $A$, we denote by $S_{a}$ and $P_{a}$, respectively, the corresponding simple and the corresponding projective $A$-modules. If, actually, $a \in C_{0}$, we denote by $S_{a}^{\prime}$, and $P_{a}^{\prime}$ the corresponding simple and the corresponding projective $C$-modules.

Proposition 1.1. Let $A$ be an algebra, $C$ be a full convex subcategory of $A$, and $P=\underset{c \in C_{0}}{\bigoplus} P_{c}$. Then, for any $c \in C_{0}$, a minimal projective resolution

$$
\ldots \rightarrow P_{n} \rightarrow \ldots \rightarrow P_{1} \rightarrow P_{0} \rightarrow S_{c} \rightarrow 0
$$

of $S_{c}$ in $\bmod A$ induces a projective resolution

$$
\ldots \rightarrow \operatorname{Hom}_{A}\left(P, P_{n}\right) \rightarrow \ldots \rightarrow \operatorname{Hom}_{A}\left(P, P_{1}\right) \rightarrow \operatorname{Hom}_{A}\left(P, P_{0}\right) \rightarrow S_{c}^{\prime} \rightarrow 0
$$

of $S_{c}^{\prime}$ in $\bmod C$. In particular, gl.dim. $C \leq \mathrm{gl} . \operatorname{dim} . A$.

Proof. Since the functor $\operatorname{Hom}_{A}(P,-)$ is exact, and, clearly, $\operatorname{Hom}_{A}\left(P, S_{c}\right) \cong S_{c}^{\prime}$, we just have to show that, for each $a \in A_{0}$ such that $P_{a}$ is a summand of one of the $P_{i}$ above, the $C$-module $\operatorname{Hom}_{A}\left(P, P_{a}\right)$ is projective.

First, if $a \in C_{0}$, then, by [4, II.2.1], $\operatorname{Hom}_{A}\left(P, P_{a}\right) \cong P_{a}^{\prime}$. Next, assume $a \notin C_{0}$. We claim that, if $P_{a}$ is a summand of one of the $P_{i}$ above, then $\operatorname{Hom}_{A}\left(P, P_{a}\right)=0$. Indeed, if this is not the case, there exists $d \in C_{0}$ such that $\operatorname{Hom}_{A}\left(P_{d}, P_{a}\right) \neq 0$. Hence, we have a path $a \rightarrow \ldots \rightarrow d$ in $A$. However, $P_{a}$ being a summand of one of the $P_{i}$ implies the existence of a sequence of morphisms $P_{a} \rightarrow \ldots \rightarrow P_{c}=P_{0}$, hence a path $c \rightarrow \ldots \rightarrow a$ in $A$. Thus, we have a path $c \rightarrow \ldots \rightarrow a \rightarrow \ldots \rightarrow d$ in $A$, with $c d \in C_{0}$. The convexity of $C$ implies $a \in C_{0}$, a contradiction.

We shall apply this proposition to the following situation. Let $\Lambda$ be an algebra. A cycle in $\bmod \Lambda$ is a sequence of non-zero non-isomorphisms $X=X_{0} \stackrel{f_{1}}{\rightarrow} X_{1} \stackrel{f_{3}}{\rightarrow} \ldots \stackrel{f}{\rightarrow} X_{t}=X$ where $t \geq 1$ and the $X_{i}$ are indecomposable $\Lambda$-modules. An algebra $\Lambda$ is called representation-directed if $\bmod \Lambda$ contains no cycle. For instance, a representation-finite simply connected algebra is always representation-directed. By $[14$, p. 78] a representation-directed algebra is always representation-finite. Moreover, it is clearly 
triangular (that is, its ordinary quiver contains no oriented cycle), and its AuslanderReiten quiver $\Gamma(\bmod \Lambda)$ is postprojective as a translation quiver (hence, by $[14$, p. 63], is standard).

Let $A$ be the Auslander algebra of a representation-directed algebra $\Lambda$, and $C$ a full convex subcategory of $A$. Since $\Gamma(\bmod \Lambda)$ is standard, then, as locally bounded $k$-linear categories, $A$ and the mesh category of $\Gamma(\bmod \Lambda)$ are equivalent. In particular, $C$ can be identified with a full convex subcategory of $\Gamma(\bmod \Lambda)$. Let $N$ be the direct sum of a complete set of representatives of the isomorphism classes of the indecomposables lying in $C$. Then $C=\operatorname{End}_{\Lambda} N$. If $X \in C_{0}$, then the corresponding projective $C$-module is $P_{X}^{\prime}=\operatorname{Hom}_{\Lambda}(N, X)$. Since End $_{\Lambda} X \cong k$, the corresponding simple $C$-module is $S_{X}^{\prime} \cong$ End $_{\Lambda} X$, and a projective cover is given by the morphism $\operatorname{Hom}_{\Lambda}(j, X): \operatorname{Hom}_{\Lambda}(N, X) \rightarrow$ End $_{\Lambda} X$, where $j: X \rightarrow N$ is the canonical section. The following statement is a straightforward generalisation of the well-known description of projective resolutions over Auslander algebras [3, III.4].

COROLlaRY 1.2. With the above notation, let $\left(g, g^{\prime}\right): Y \oplus Y^{\prime} \rightarrow X$ be a right minimal almost split morphism in $\bmod \Lambda$ where $X$ and the indecomposable summands of $Y$ are in $C$, but the indecomposable summands of $Y^{\prime}$ are not in $C$. Let $j: X \rightarrow N$ be the canonical section.

(a) If $\tau X \notin C_{0}$, then the sequence

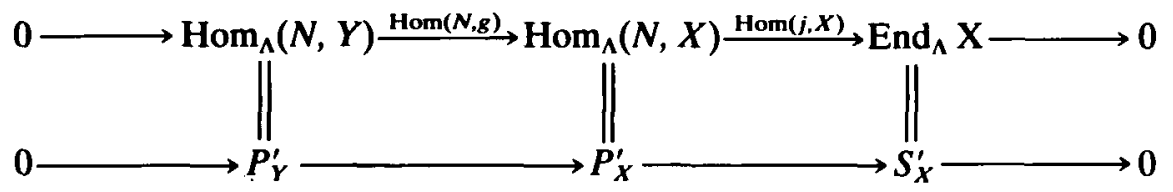

is a minimal projective resolution of $S_{X}^{\prime}$ in $\bmod C$

(b) If $\tau X \in C_{0}$, then $Y^{\prime}=0$, and the almost split sequence $0 \rightarrow \tau X \stackrel{f}{\rightarrow} Y \stackrel{s}{\rightarrow} X \rightarrow 0$ induces an exact sequence

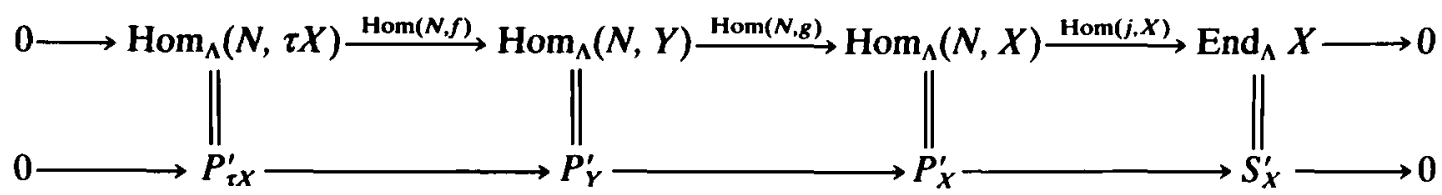

which is a minimal projective resolution of $S_{X}^{\prime}$ in $\bmod C$.

Proof. (a) Consider the simple $A$-module $S_{X}$. By [3, III.4], if $X$ is not a projective $\Lambda$-module, and the almost split sequence ending at $X$ is $0 \rightarrow \tau X \stackrel{\left(\begin{array}{l}f \\ f^{\prime}\end{array}\right)}{\longrightarrow} Y \oplus Y^{\prime} \stackrel{\left(g, g^{\prime}\right)}{\longrightarrow}$ $X \rightarrow 0$, then $S_{X}$ has a minimal projective resolution in $\bmod A$ of the form

$0 \rightarrow \operatorname{Hom}_{\Lambda}(M, \tau X) \stackrel{\operatorname{Hom}\left(M\left(f^{\prime}\right)\right)}{\longrightarrow} \operatorname{Hom}_{\Lambda}(M, Y)$

$$
\oplus \operatorname{Hom}_{\Lambda}\left(M, Y^{\prime}\right) \stackrel{\operatorname{Hom}\left(M \cdot\left(g, g^{\prime}\right)\right)}{\longrightarrow} \operatorname{Hom}_{\Lambda}(M, X) \rightarrow S_{X} \rightarrow 0
$$


We apply the functor $\operatorname{Hom}_{A}\left(\operatorname{Hom}_{\Lambda}(M, N),-\right)$ and obtain, by (1.1), a projective resolution of $S_{X}^{\prime}$ in $\bmod C$. Since the hypothesis implies that $\operatorname{Hom}_{\Lambda}(N, \tau X)=$ $\operatorname{Hom}_{\Lambda}\left(N, Y^{\prime}\right)=0$, this gives, by [4, II.2.1], the required projective resolution. Clearly, it is minimal. The proof is similar if $X$ is a projective $\Lambda$-module.

(b) Since $X$ and $\tau X$ belong to $C$, which is convex, it follows that $Y^{\prime}=0$. The proof then proceeds as in (a).

We now show that if $X$ is an indecomposable $A$-module in $C$ such that $\tau X$ is also in $C$, then the $C$-module rad $P_{X}$ is indecomposable.

LEMma 1.3. If $X$ and $\tau X$ are in $C$, and $0 \rightarrow \tau X \stackrel{f}{\rightarrow} Z \stackrel{g}{\rightarrow} X \rightarrow 0$ is an almost split sequence, then

$$
\operatorname{End}_{C}\left(\operatorname{rad} P_{X}\right) \cong\left\{u \in \operatorname{End}_{\wedge} Y \mid g u f=0\right\} \cong k
$$

In particular, $\operatorname{rad} P_{X}$ is indecomposable.

Proof. By (1.2)(b), we have a short exact sequence $0 \rightarrow P_{\tau X} \stackrel{\operatorname{Hom}(N, f)}{\longrightarrow}$ $P_{Y} \stackrel{\operatorname{Hom}(N, g)}{\longrightarrow} \operatorname{rad} P_{X} \rightarrow 0$. By applying respectively the exact functor $\operatorname{Hom}_{C}(P,-)$ and the left exact functor $\operatorname{Hom}_{C}\left(-, \operatorname{rad} P_{X}\right)$, we obtain the exact sequences

$$
0 \rightarrow \operatorname{Hom}_{C}\left(P_{Y}, P_{\tau X}\right) \rightarrow \operatorname{Hom}_{C}\left(P_{Y}, P_{Y}\right) \stackrel{\operatorname{Hom}\left(P_{Y}, \operatorname{Hom}(N, g)\right)}{\longrightarrow} \operatorname{Hom}_{C}\left(P_{Y}, \operatorname{rad} P_{X}\right) \rightarrow 0
$$

and

$0 \rightarrow \mathrm{Hom}_{C}\left(\operatorname{rad} P_{X}, \operatorname{rad} P_{X}\right) \rightarrow$

$$
\operatorname{Hom}_{C}\left(P_{Y}, \operatorname{rad} P_{X}\right) \stackrel{\operatorname{Hom}_{C}\left(\operatorname{Hom}_{1}(N f), \operatorname{rad} P_{X}\right)}{\longrightarrow} \operatorname{Hom}_{C}\left(P_{\tau X}, \operatorname{rad} P_{X}\right)
$$

In the first exact sequence, we have $\operatorname{Hom}_{C}\left(P_{Y}, P_{\tau X}\right) \cong \operatorname{Hom}_{\Lambda}(Y, \tau X) \cong 0$, where the isomorphism follows from the hypotheses that $\Lambda$ is representation-directed and $\operatorname{Hom}_{\Lambda}(\tau X, Y) \neq 0$. Thus $\operatorname{Hom}_{C}\left(P_{Y}, \operatorname{Hom}_{\Lambda}(N, g)\right)$ is an isomorphism, and $\operatorname{Hom}_{C}\left(P_{Y}, \operatorname{rad} P_{X}\right) \cong \operatorname{Hom}_{C}\left(P_{Y}, P_{Y}\right) \cong \operatorname{End}_{\Lambda} Y$. The image of $u$ in $\operatorname{End}_{\Lambda} Y$ under this isomorphism is easily checked to be $\operatorname{Hom}_{\Lambda}(N, g u): P_{Y} \rightarrow \operatorname{rad} P_{X}$. From the second exact sequence above, we deduce

$$
\begin{aligned}
\operatorname{End}_{C}\left(\operatorname{rad} P_{X}\right) & \cong \operatorname{Ker}_{\operatorname{Hom}}\left(\operatorname{Hom}_{\Lambda}(N, g), \operatorname{rad} P_{X}\right) \\
& \cong\left\{v: P_{Y} \rightarrow \operatorname{rad} P_{X} \mid v \operatorname{Hom}_{\Lambda}(N, f)=0\right\} \\
& \cong\left\{u \in \operatorname{End}_{\Lambda} Y \mid \operatorname{Hom}_{\Lambda}(N, g u) \operatorname{Hom}_{\Lambda}(N, f)=0\right\} \\
& \cong\left\{u \in \operatorname{End}_{\Lambda} Y \mid \operatorname{Hom}(N, g u f)=0\right\} \\
& \cong\left\{u \in \operatorname{End}_{\Lambda} Y \mid g u f=0\right\} .
\end{aligned}
$$

It remains to show that $\operatorname{End}_{C}\left(\operatorname{rad} P_{X}\right) \cong k$. For this purpose, we shall show that every non-zero $u \in \operatorname{End}_{\Lambda} Y$ satisfying $g u f=0$ is an isomorphism. We first note that these hypotheses imply $g u \neq 0$. For, if $g u=0$, there exists, by the universal property of kernels, a morphism $u^{\prime}: Y \rightarrow \tau X$ such that $u=f u^{\prime}$. Since $\Lambda$ is representation-directed and $\operatorname{Hom}_{\Lambda}(\tau X, Y) \neq 0$, we have $u^{\prime}=0$, hence $u=0$, a contradiction. Thus, $g u f=0$ with 
$g u \neq 0$ implies, by the universal property of cokernels, the existence of a non-zero morphism $w: X \rightarrow X$ such that $w g=g u$. Since $\Lambda$ is representation-directed, we have End $_{\Lambda} X \cong k$ and hence $w$ must be an isomorphism. Since $w^{-1} g: Y \rightarrow X$ is not a retraction, there exists $u^{\prime}: Y \rightarrow Y$ such that the following diagram is commutative.

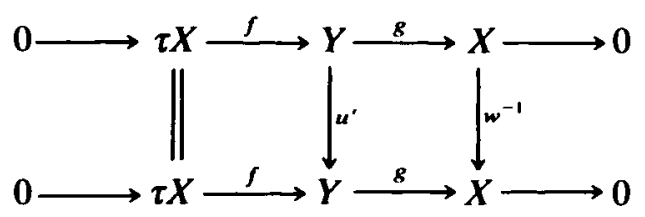

Consequently, $g\left(u u^{\prime}\right)=(g u) u^{\prime}=(w g) u^{\prime}=w\left(w^{-1} g\right)=g$. Since $g$ is right minimal, $u u^{\prime}$ is an isomorphism. Similarly, $u^{\prime} u$ is also an isomorphism. Therefore, so is $u$. Thus, End $_{C}\left(\operatorname{rad} P_{X}\right)$ is a division ring which is finite dimentional over the algebraically closed field $k$. Hence End $_{C}\left(\operatorname{rad} P_{X}\right) \cong k$.

2. Proof of the main theorem. As pointed out in the introduction, we only need to show that (a) implies (c), and that (b) implies (a).

In order to show that (a) implies (c), we use the following strategy. We first recall that, by $[15,(4.1)]$, a triangular algebra $A$ is strongly simply connected if each full convex subcategory $C$ of $A$ satisfies the separation condition of [5]. Let thus $\Lambda$ be a simply connected representation-finite algebra. Then $\Lambda$ is representation-directed. By (1.3), if $C$ is a full convex subcategory of the Auslander algebra $A$ of $\Lambda$, and $X$ is an indecomposable module in $C$ such that $\tau X$ is also in $C$, then $\operatorname{rad} P_{X}$ is indecomposable. That is, in this case, $\operatorname{rad} P_{X}$ is trivially separated. Thus, in order to show that $A$ is strongly simply connected, it suffices to check that $\operatorname{rad} P_{X}$ is separated whenever $X$ is in $C$ and $\tau X$ is not in C.

For this purpose, we need the orbit quiver $\sigma_{\Lambda}$ of $\Lambda$ (see [5] or $[14, p .49]$. Recall that, if $\Lambda$ is representation-directed, then $O_{\Lambda}$ is a quiver defined as follows; a point in $O_{\Lambda}$ is the $\tau$-orbit $\mathscr{O}(X)$ of an indecomposable $\Lambda$-module $X$, and there is an arrow $\mathscr{O}(X) \rightarrow \mathscr{O}(Y)$ if and only if there is an arrow $\tau^{m} X \rightarrow P$ in $\Gamma(\bmod \Lambda)$, where $m \geq 0$, and $P$ is the unique indecomposable projective $\Lambda$-module in the $\tau$-orbit of $Y$. It is well known that $\Lambda$ is simply connected if and only if $\hat{O}_{\Lambda}$ is a tree (see [7, (6.5)] or [12, (4.13)] and [5, (2.2]).

Lemma 2.1. Let $\Lambda$ be a representation-finite simply connected algebra, and $A$ the Auslander algebra of $\Lambda$. Then $A$ is strongly simply connected.

Proof. Let $C$ be a full convex subcategory of $A$. It follows from the above discussion that we only need to show that $\operatorname{rad} P_{X}$ is separated whenever $X$ is in $C$, while $\tau X$ is not in C. By (1.2)(a), $\operatorname{rad} P_{X}$ is projective. Let $P_{U}$ and $P_{U}$, be distinct indecomposable summands of $\operatorname{rad} P_{X}$. Since $\Lambda$ is representation-finite, we have $\operatorname{dim}_{k} \operatorname{Irr}(U, X)=\operatorname{dim}_{k} \operatorname{Irr}\left(U^{\prime}, X\right)=1$, where $\operatorname{Irr}\left(X_{1}, X_{2}\right)$ denotes the space of irreducible morphisms from $X_{1}$ to $X_{2}$. Therefore $U$ and $U^{\prime}$ are not isomorphic. Assume $P_{V}$ lies in the support of $P_{U}$, and $P_{V}$ lies in the support of $P_{U^{\prime}}$. Then $\operatorname{Hom}_{\Lambda}(U, V) \cong \operatorname{Hom}_{C}\left(P_{U}, P_{V}\right) \neq 0$, and $\operatorname{Hom}_{\Lambda}\left(U^{\prime}, V^{\prime}\right) \cong$ $\operatorname{Hom}_{C}\left(P_{U^{\prime}}, P_{V^{\prime}}\right) \neq 0$, so that we have paths in $C$ of the forms $U \rightarrow \ldots \rightarrow V$ and $U^{\prime} \rightarrow \ldots \rightarrow V^{\prime}$. These paths correspond to walks of the forms $\mathcal{O}(U)-\ldots-\mathcal{O}(V)$ and $\mathcal{O}\left(U^{\prime}\right)-\ldots-\mathcal{O}\left(V^{\prime}\right)$ in $O_{\Lambda}$. If $\operatorname{rad} P_{X}$ is not separated, there is a walk $V-W_{1}-W_{2}-\ldots-$ $W_{t}-V^{\prime}$ in $C$ such that no point on the walk is the source of a path ending at $X$. If some 
point $W_{i}$ on this walk were in the $\tau$-orbit of $X$, we would have a path in the quiver of $C$ of the form

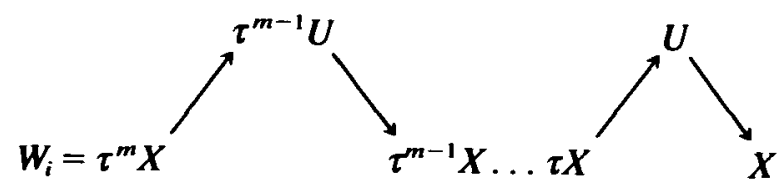

with $m \geq 1$, and this would imply by convexity that $\tau X$ lies in $C$, a contradiction. Hence no $W_{i}$ lies in the $\tau$-orbit of $X$. Now, we have a walk in $\sigma_{\Lambda}$ of the form

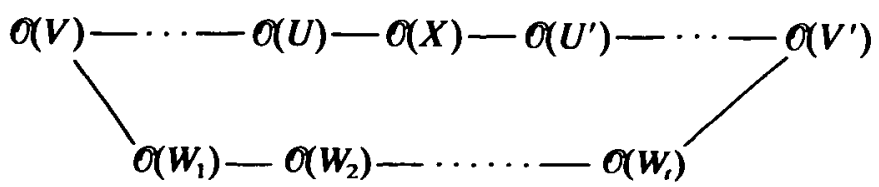

which is a non-trivial cyclic walk in $\mathcal{O}_{\Lambda}$. This contradicts the fact that $\mathcal{O}_{\Lambda}$ is a tree and shows that $\operatorname{rad} P_{X}$ is separated. Consequently, $C$ is separated and $A$ is strongly simply connected.

In order to show that (b) implies (a), we shall use the fact that, by [13, (4.3)], a representation-finite algebra $\Lambda$ is simply connected if and only if the fundamental group of the Auslander-Reiten quiver $\Gamma(\bmod \Lambda)$, as defined in [7], is trivial. The following lemma completes the proof of the main theorem.

Leмma 2.2. Let $\Lambda$ be a representation-finite algebra such that its Auslander algebra $A$ is simply connected. Then $\Lambda$ is simply connected.

Proof. Clearly, the simple connectedness of $A$ implies that $A$ is representationdirected. As pointed out before, this implies that $\Gamma(\bmod \Lambda)$ is standard. That is, we have a bound quiver presentation $A \cong k \Gamma(\bmod \Lambda) / \mathcal{M}$, where $k \Gamma(\bmod \Lambda)$ is the path algebra of $\Gamma(\bmod \Lambda)$, and $\mathcal{M}$ is the ideal of $k \Gamma(\bmod \Lambda)$ generated by the mesh relations. Now it is easily seen that the homotopy relations defining the fundamental groups of the Auslander-Reiten quiver $\Gamma(\bmod \Lambda)$, as defined in [7], and of the bound quiver presentation $(\Gamma(\bmod \Lambda), \mathcal{M})$ of $A$ coincide. Since $A$ is simply connected, the latter vanishes. Hence, so does the first, that is, $\Lambda$ is simply connected.

We conclude this paper with the following corollary.

COROLlARY 2.3. Let $\Lambda$ be a representation-finite simply connected algebra, and $C$ a full convex subcategory of the Auslander algebra of $\Lambda$. Then $C$ has a postprojective component.

Proof. Indeed, this follows from the fact that $C$ satisfies the separation condition (by our theorem) hence, by [6], has a postprojective component.

ACKNOwledgements. This work was done while the second author was a postdoctoral fellow of the Ministère de l'Enseignement Supérieur et de la Science du Québec. The first author gratefully acknowledges partial support from the Natural Sciences and 
Engineering Research Council of Canada. The authors would also like to thank Dan Zacharia for a helpful discussion.

\section{REFERENCES}

1. I. Assem and A. Skowroniski, On some classes of simply connected algebras, Proc. London Math. Soc. (3) 56 (1988), 417-450.

2. I. Assem and J. A. de la Peña, The fundamental groups of a triangular algebra, Comm. Algebra 24 (1) (1996), 187-208.

3. M. Auslander, Representation dimension of artin algebras, Queen Mary College Mathematics Notes (1971), London.

4. M. Auslander, I. Reiten and S. Smalø, Representation theory of Artin algebras, Cambridge Studies in Advanced Mathematics 36 (Cambridge University Press, 1994).

5. R. Bautista, F. Larrión, and L. Salmerón, On simply connected algebras, J. London Math. Soc. (2) 27 (1983), 212-220.

6. K. Bongartz, A criterion for finite representation type, Math. Ann. 269 (1984), 1-12.

7. K. Bongartz and P. Gabriel, Covering spaces in representation-theory, Invent. Math. 65 (1982), 331-378.

8. O. Bretscher and P. Gabriel, The standard form of a representation-finite algebra, Bull. Soc. Math. France 111 (1983), 21-40.

9. H. Cartan and S. Eilenberg, Homological algebra (Princeton University Press, 1956).

10. D. Happel, Hochschild cohomology of finite-dimensional algebras, Séminaire d'Algèbre $P$. Dubreil et M.-P. Malliavin (Paris, 1987-1988), Lecture Notes in Mathematics 1404 (Springer, 1989), 108-126.

11. D. Happel, Hochschild cohomology of Auslander algebras, Topics in Algebra, Banach Center Publ. 26 part 1 (PWN, 1990), 303-310.

12. F. Larrión, Algebras forestales, Ph.D. Thesis, Universidad Nacional Autónoma de México, 1981.

13. R. Martínez-Villa and J. A. de ra Peña, The universal cover of a quiver with relations, $J$. Pure Appl. Algebra 30 (1983), 277-292.

14. C. M. Ringel, Tame algebras and integral quadratic forms, Lecture Notes in Mathematics, 1099 (Springer, 1984).

15. A. Skowroński, Simply connected algebras and Hochschild cohomologies, Proceedings of the Sixth International Conference on Representations of Algebras (Ottawa, 1992), Canad. Math. Soc. Proc. 14 (1993), 431-437.

Département de Mathématiques et d INFormatique

UNIVERSITÉ DE SHERBROOKE

SHERBROOKE (QuÉBEC) J1K 2R1

CANADA

Current address of Peter Brown:

169-I BritTany Manor Drive

AMHERST, MA 01002

U.S.A. 